\title{
(Quantum) Space-Time as a Statistical Geometry of Fuzzy Lumps and the Connection with Random Metric Spaces
}

\author{
Manfred Requardt \\ (email: requardt@theorie.physik.uni-goettingen.de) \\ Institut für Theoretische Physik \\ Universität Göttingen \\ Bunsenstrasse 9 \\ 37073 Göttingen Germany \\ and \\ Sisir Roy \\ (email:sisir@isical.ac.in ) \\ Indian Statistical Institute \\ Calcutta -700 035 \\ India
}

\begin{abstract}
We develop a kind of pregeometry consisting of a web of overlapping fuzzy lumps which interact with each other. The individual lumps are understood as certain closely entangled subgraphs (cliques) in a dynamically evolving network which, in a certain approximation, can be visualized as a time-dependent random graph. This strand of ideas is merged with another one, deriving from ideas, developed some time ago by Menger et al, that is, the concept of probabilistic- or random metric spaces, representing a natural extension of the metrical continuum into a more microscopic regime. It is our general goal to find a better adapted geometric environment for the description of microphysics. In this sense one may it also view as a dynamical randomisation of the causal-set framework developed by e.g. Sorkin et al. In doing this we incorporate, as a perhaps new aspect, various concepts from fuzzy set theory.
\end{abstract}




\section{Introduction}

There exists a certain suspicion among the scientific community that nature may be discrete or rather "behaves discretely" on the Planck scale. But even if one is willing to agree with this "working philosophy", it is far from being evident what this vague metaphor actually means or how it should be implemented into a concrete and systematic inquiry concerning physics and mathematics in the Planck regime.

There are basically two overall attitudes as to "discreteness on the Planck scale", the one starts (to a greater or lesser degree) from continuum concepts (or more specifically: concepts being more or less openly inspired by them) and then try to detect or create modes of "discrete behavior" on very fine scales, typically by imposing quantum theory in full or in parts upon the model system or framework under discussion. We call it "top down" approach.

There are prominent and very promising candidates in this class like e.g.string theory or loop quantum gravity. Spin network is a more recent version (or rather: aspect) of the latter approach. As these approaches are meanwhile widely known we refrain from citing from the vast corresponding literature. We recommend instead as more recent reviews as to the latter approach, containing some cursory remarks about the former together with a host of references, [1] and [2] and, as a beautiful introduction to the conceptual problems of quantum gravity in general, [3].

In the following we undertake to describe how macroscopic space-time (or rather, its underlying mesoscopic or microscopic substratum) is supposed to emerge as a superstructure of a web of lumps in a fluctuating dynamical cellular network. We call this the "bottom up" approach. In doing this, two strands of research are joined, which, originally, started from different directions. The one is the cellular network and random graph approach, developed by one of the authors (M.R.), the other the statistical geometry of lumps, a notion originally coined by Menger and coworkers and being further developed by various groups (see e.g. [16], [17, [18], [19]). It is worth mentioning that Einstein himself was not against such a grainy substratum underlying our space-time continuum (see the essay of Stachel in [20]).

The point where these different strands meet is the following. In the dynamical network approach of (M.R.) macroscopic space-time is considered to be a coarsegrained emergent phenomenon (called an orderparameter manifold in [8]). It is assumed to be the result of some kind of geometric phase transition (very much in the spirit of the physics of self-organisation). This framework was developed in quite some detail in e.g. [8]. We argued there that, what we consider to be the elementary building blocks of continuous space-time, i.e. the so-called physical points, are on a finer scale actually densely entangled subclusters of nodes and bonds of the underlying network or graph. In [8] we called them also cliques (which denote in graph theory the maximal complete subgraphs or 
maximal subsimplices of a given graph).

We further argued there that the substructure of our space-time manifold consists in fact of two stories, the primordial network, dubbed by us $Q X$, and, overlying it, the web of lumps or cliques, denoted by $S T$, which can also be viewed as a coarser mesoscopic network with the cliques or lumps as supernodes and with superbonds which connect lumps having a non-void overlap. This correrspondence raises the possibility to relate the lumps or cliques of [8] with the lumps occurring in the approach of Menger et al.

One should however note that the two philosophies are not entirely the same. In [8] and related work the lumps emerge from a more primordial discrete dynamical substratum, with the lumps having a specific internal structure. In the approach of Menger et al (at least as far as we can see) they figure as the not further resolvable building blocks of space-time if one approaches the so-called Planck-regime from above, i.e. from the continuum side. In other words, the former approach is more bottom-up oriented while the latter one is more top-down. About such not further resovable scales of space-time (where the ordinary continuum picture ends) was of course also speculated by quite a few other people, most notably Wheeler. Our personal working philosophy is that space-time at the very bottom (i.e. near or below the notorious Planck scale) resembles or can be modeled as an evolving information processing cellular network, consisting of elementary modules (with, typically, simple internal discrete state spaces) interacting with each other via dynamical bonds which transfer the elementary pieces of information among the nodes. That is, the approach shares the combinatorial point of view in fundamental space-time physics which has been advocated by e.g. Penrose. It is a crucial and perhaps characteristic extra ingredient of our framework that the bonds (i.e. the elementary interactions) are not simply dynamical degrees of freedom (as with the nodes their internal state spaces are assumed to be simple) but can a fortiori, depending on the state of the local network environment, be switched on or off, i.e. can temporarily be active or inactive! This special ingredient of the dynamics hopefully allows the network to perform geometric phase transitions into a new ordered phase displaying a certain two-story structure to be explained below. This conjectured emergent geometric order can be viewed as kind of a discrete proto space-time or pregeometry carrying metrical, causal and dimensional structures (as to the cellular network approach cf. [4] to [8], the other point of view is expounded in e.g. the book of Roy; 18 to which we also refer for a more complete list of references).

We will see that several types of distance concepts do emerge in our analysis on the various scales of resolution of space-time. On the in our scheme most fundamental level, that is, the primordial network (or graph), we have a natural distance concept (node distance). The same holds for the next fundamental level, that is, the network of lumps (the clique graph; see below). If we leave these fundamental levels (with their relatively discrete behavior) and enter the realm of quasi-continuity, other possibilities do emerge. It is interesting to relate these 
various concepts to each other and to distance concepts discussed e.g. in [19] or [38]. We show in particular that our network of fuzzy lumps leads naturally to the concept of random metric spaces (see section (7.2)). Spaces of this kind should emerge on the interface between the presumed discrete Planck scale scenario and a perhaps quasi-continuous, that is, coarse-grained spatial environment, connecting this primordial level with the ordinary continuous regime.

One of the aims of our paper is to relate these various strands of ideas to each other. Our overall working philosophy is that geometry has to emerge from a purely relational picture. We want to reconstruct the continuum concepts of ordinary physics and/or mathematics from more primordial pregeometric (and basically discrete) ones which, in our view, hold sway on the Planck scale. This may then shed some light on the better understanding of quantum mechanics as well as on the concurrent top-down approaches mentioned above. We note in passing, that this point of view has a venerable history of its own, beginning with e.g. Leibniz. In the following we undertake to randomize metrical concepts. A related but technically different approach was developed by Isham et al some time ago (see e.g. [21] or [22]), who chose to quantise metrical concepts. It would be an interesting task to relate these two approaches to each other.

Another precursor may be the causal-set approach of Sorkin et al (see e.g. [29] or [30]). In a sense our framework adds dynamics, viz. interaction, and randomisation to this picture.

\section{The Cellular Network Environment}

Motivated by the above working philosophy, we emulate the underlying substratum of our world, or, more specifically, of our space-time (quantum) vacuum (containing however in addition all the existing quantum and macro objects as extended excitation patterns!) by what we call a cellular network. This discrete structure consists of elementary nodes, $n_{i}$, which interact (or exchange information) with each other via bonds, $b_{i k}$, playing the role of (in this context) not further reducible elementary interactions. The possible internal structure of the nodes (modules) or bonds (interaction chanels) is emulated by discrete internal state spaces carried by the nodes/bonds. The node set is assumed to be large but finite or countable. The bond $b_{i k}$ is assumed to connect the nodes $n_{i}, n_{k}$. The internal states of the nodes/bonds are denoted by $s_{i}, J_{i k}$ respectively. As our philosophy is, to generate complex behavior out of simple models we, typically, make simple choices for them, one being e.g.

$$
s_{i} \in q \cdot \mathbb{Z} \quad, \quad J_{i k} \in\{-1,0,+1\}
$$

with $q$ an elementary quantum of information.

As in our approach the bond states are dynamical degrees of freedom which, a fortiori, can be switched off or on, the wiring, that is the pure geometry of the 
network is also an emergent, dynamical property and is not given in advance. Consequently, the nodes and bonds are typically not arranged in a more or less regular array, a lattice say, with a fixed nea-/far-order. This implies that geometry will become to some extent a relational (Machian) concept and is no longer an ideal element (cf. the more detailed discussion in [11], which deals primarily with the emergence of quantum theory as a consequence of the geometric fine structure of such a network).

On the other side, as in cellular automata, the node and bond states are updated (for convenience) in discrete clock time steps, $t=z \cdot \tau, z \in \mathbb{Z}$ and $\tau$ being an elementary clock time interval. This updating is given by some local dynamical law (examples given below). In this context local means that the node/bond states are changed at each clock time step according to a prescription with input the overall state of a certain neighborhood (in some topology) of the node/bond under discussion. We want however to emphasize that $t$ is not to be confounded with some physical time, which, for its part, is also considered to be an emergent coarse grained quantity. The well known problem of time is, for the time being, not treated in detail in the following, as it presents a big problem of its own, needing a careful and separate analysis (see e.g. [9] or [10]). That is, at the moment the above clock time is neither considered to be dynamical nor observer dependent. We discussed however the presumed emergence of a new primordial time scale which sets the scale for the regime where quantum fluctuations hold sway in [11].

A simple example of such a local dynamical law we are having in mind is given in the following definition.

Definition 2.1 (Example of a Local Law) At each clock time step a certain quantum $q$ is exchanged between, say, the nodes $n_{i}, n_{k}$, connected by the bond $b_{i k}$ such that

$$
s_{i}(t+\tau)-s_{i}(t)=q \cdot \sum_{k} J_{k i}(t)
$$

(i.e. if $J_{k i}=+1$ a quantum $q$ flows from $n_{k}$ to $n_{i}$ etc.)

The second part of the law describes the back reaction on the bonds (and is, typically, more subtle). This is the place where the so-called hysteresis interval' enters the stage. We assume the existence of two 'critical parameters' $0 \leq \lambda_{1} \leq$ $\lambda_{2}$ with:

$$
\begin{gathered}
J_{i k}(t+\tau)=0 \quad \text { if } \quad\left|s_{i}(t)-s_{k}(t)\right|=:\left|s_{i k}(t)\right|>\lambda_{2} \\
J_{i k}(t+\tau)= \pm 1 \quad \text { if } \quad 0< \pm s_{i k}(t)<\lambda_{1}
\end{gathered}
$$

with the special proviso that

$$
J_{i k}(t+\tau)=J_{i k}(t) \quad \text { if } \quad s_{i k}(t)=0
$$


On the other side

$$
J_{i k}(t+\tau)=\left\{\begin{array}{ll} 
\pm 1 & J_{i k}(t) \neq 0 \\
0 & J_{i k}(t)=0
\end{array} \quad \text { if } \quad \lambda_{1} \leq \pm s_{i k}(t) \leq \lambda_{2}\right.
$$

In other words, bonds are switched off if local spatial charge fluctuations are too large, switched on again if they are too small, their orientation following the sign of local charge differences, or remain inactive.

Another interesting law arises if one exchanges the role of $\lambda_{1}$ and $\lambda_{2}$ in the above law, that is, bonds are switched off if the local node fluctuations are too small and are switched on again if they exceed $\lambda_{2}$. We emulated all these laws on a computer and studied a lot of network properties. The latter law has the peculiar feature that it turned out to have very short transients in the simulations, i.e. it reaches an attractor in a very short clock time. Furthermore these attractors or state-cycles turned out to be very regular, that is, they had a very short period of typically six, that is, the whole network returned in a previous state after only six clock time steps, which is quite remarkable, given the seeming complexity of the evolution and the huge phase space ([19]).

Remarks:

1. It is important that, generically, such laws, as introduced above, do not lead to a reversible time evolution, i.e. there will typically exist attractors or state-cycles in total phase space (the overall configuration space of the node and bond states). On the other hand, there exist strategies (in the context of cellular automata!) to design particular reversible network laws (cf. e.g. [13]) which are, however, typically of second order. Usually the existence of attractors is considered to be important for pattern formation. On the other side, it may suffice that the phase space, occupied by the system, shrinks in the course of evolution, that is, that one has a flow into smaller subvolumes of phase space.

2. In the above class of laws a direct bond-bond interaction is not yet implemented. We are prepared to incorporate such a (possibly important) contribution in a next step if it turns out to be necessary. In any case there are not so many ways to do this in a sensible way. Stated differently, the class of possible physically sensible interactions is perhaps not so numerous.

3. As in the definition of evolution laws of spin networks by e.g. Markopoulou, Smolin and Borissov (see [14] or [15]), there are in our case more or less two possibilities: treating evolution laws within an integrated space-time formalism or regard the network as representing space alone with the time evolution being implanted via some extra principle ( which is the way we have chosen above). The interrelation of these various approaches and 
frameworks, while being very interesting, is however far from obvious at the moment and needs a separate detailed investigation.

Observation 2.2 (Gauge Invariance) The above dynamical law depends nowhere on the absolute values of the node charges but only on their relative differences. By the same token, charge is nowhere created or destroyed. We have

$$
\Delta\left(\sum_{Q X} s(n)\right)=0
$$

To avoid artificial ambiguities we can e.g. choose a fixed reference level and take as initial condition respectively constraint

$$
\sum_{Q X} s(n)=0
$$

There are many different aspects of our class of cellular networks one can study in this context. One can e.g. regard them as complex dynamical systems, or one can undertake to develop a statistical or stochastic framework etc. In a purely geometric sense, however, they are evolving graphs. As we are in this paper primarily concerned with the analysis of the microstructure of (quantum) space-time, it seems to be a sensible strategy to supress, at least in a first step, all the other features like e.g. the details of the internal state spaces of nodes and bonds and concentrate instead on their pure wiring diagram and its reduced (graph) dynamics. This is already an interesting characteristic of the network (perhaps somewhat reminiscent of the Poincaré map in the theory of chaotic systems) as bonds can be switched on and off in the course of clock time so that already the wiring diagram will constantly change. Furthermore, as we will see, it encodes the complete near-and far-order structure of the network, that is, it tells us which regions are experienced as near by or far away (in a variety of possible physical ways such as strength of correlations or with respect to some other physically meaningful metric like e.g. statistical distance etc.). Evidently this is one of the crucial features we expect from something like physical spacetime. In the above simple scenario with $J_{i k}= \pm 1$ or 0 one can e.g. draw a directed bond, $d_{i k}$, if $J_{i k}=+1$, with $J_{i k}=-J_{k i}$ implied, and delete the bond if $J_{i k}=0$. This leads to a (clock) time dependent graph, $G(t)$, or wiring diagram. In other words, we will deal in the following mainly with the evolution and structure of large dynamical graphs.

We close this section with a brief résumé of the characteristics an interesting network dynamics should encode (in our view).

Résumé 2.3 Irrespectively of the technical details of the dynamical evolution law under discussion it should emulate the following, in our view crucial, principles, in order to match certain fundamental requirements concerning the capability of emergent and complex behavior. 
1. As is the case with, say, gauge theory or general relativity, our evolution law on the surmised primordial level should implement the mutual interaction of two fundamental substructures, put sloppily: "geometry" acting on "matter" and vice versa, where in our context "geometry" is assumed to correspond in a loose sense with the local and/or global bond states and "matter" with the structure of the node states.

2. By the same token the alluded selfreferential dynamical circuitry of mutual interactions is expected to favor a kind of undulating behavior or selfexcitation above a return to some uninteresting 'equilibrium state' as is frequently the case in systems consisting of a single component which directly acts back on itself. This propensity for the 'autonomous' generation of undulation patterns is in our view an essential prerequisite for some form of "protoquantum behavior" we hope to recover on some coarse grained and less primordial level of the network dynamics.

3. In the same sense we expect the overall pattern of switched-on and-off bonds to generate a kind of "protogravity".

\section{The Network of Lumps}

In [8] we argued that our microscopic cellular network, $Q X$, may be capable of performing a geometric phase transition into a more ordered phase, dubbed $Q X / S T$, with elementary building blocks the maximal connected subgraphs or cliques (maximal subsimplices) occurring in the primordial graph, belonging to $Q X$. We further argued that this emergent orderparameter manifold (as it plays a role similar to ordinary orderparameters in the statistical mechanics of phase transitions) may constitute a stochastic protoform of our ordinary space-time or quantum vacuum with the cliques as protoforms of physical points (lumps), having an inner dynamical structure and being entangled with the other (overlapping) lumps via common nodes or bonds.

The stochastic aspects are brought in by the underlying dynamical network law, which induces, among other things, a certain amount of creation and annihilation of bonds among the microscopic nodes. As a consequence the size and shape of the cliques or lumps fluctuates in the course of network evolution. These aspects have been analyzed in quite some detail in [8] within the framework of random graphs.

This derived coarser network, viz. the clique graph or web of lumps, is defined as follows. The cliques are represented by new meta-nodes, the respective metabonds represent overlap of cliques (in form of common nodes).

Observation 3.1 Note that, while this new network may be regarded as being coarser in some sense, it may nevertheless in general consist of much more nodes 
and bonds than the underlying primordial network. Usually there are much more maximal subsimplices than primordial nodes, as a given node will typically belong to quite a few different maximal subsimplices (cf. the estimates in [8]). This array of intersecting maximal subsimplices has the natural structure of a simplicial complex with the smaller simplices as faces of the maximal ones, viz. the cliques (as to this notion see any textbook on algebraic topology like e.g. [23], cf. also section 3.2 of [6]). If we represent this simplicial complex by a new (clique-) graph with only the maximal simplices occurring as meta-nodes we loose, on the other side, some information, as we do not keep track of, to give an example, situations where, say, three lumps or cliques have a common overlap. This situation is not distinguished from the scenario where only each pair of the triple has a common overlap.

Remark 3.2 On the other hand, it may be physically advantageous to loose microscopic information in a controlled way. We will show below that this possibility to consider the network as a simplicial complex stops at this first level, the coarser picture of a clique graph allows for a geometric renormalization group procedure as we can repeat this process on each level, thus creating a whole tower of such metagraphs, one lying over the other, leading in the end to webs which resemble more and more our ordinary space-time.

We now have to make the physically motivated assumption that our network of lumps, which is assumed to be in the phase $Q X / S T$, is not fluctuating too wildly, put differently, that the effective dynamics, induced by the underlying microscopic dynamics defined above, leaves the individual cliques, $C_{i}$, sufficiently stable. We hence assume that they do not change their form too much from clocktime step to clocktime step, so that we are able to keep track of the history of the individual cliques over an appreciable amount of time steps.

$$
C_{i}(t) \cap C_{i}(t+1) \approx C_{i}(t)
$$

This means that only a small portion of nodes enters or leaves each of the cliques in every time step. We make this assumption so as to be able to perform some sort of assemble averages over fluctuating but individual (that is, labelled) cliques in the next section and identify them with fuzzy lumps.

\section{Cliques as Fuzzy Sets}

We now concentrate on the (clock-time) evolution of an arbitrary (generic) but fixed clique, denoting its time sequence by

$$
C\left(t_{0}\right), C\left(t_{1}\right), \ldots, C\left(t_{N}\right)
$$


We argued above that the clique under observation changes its shape and size only mildly from time step to time step. As it is frequently done in physics, we want to replace the above time series of a generic clique by an ensemble. To this end we define

$$
\bar{C}:=\bigcup C\left(t_{i}\right) \quad, \quad \underline{C}:=\bigcap C\left(t_{i}\right)
$$

We now define a so-called membership function, $m(x)$, for an arbitrary node, $x$, in the graph, G. Traditionally, the usual habit in fuzzy set theory is it, to speak of fuzzy sets, but actually they do exist only via their membership functions (see below). That is, we relate to each cluster of sharp cliques, defined above, a so-called fuzzy clique, defined by its membership function. We denote the fuzzy clique, belonging to the above ensemble of sharp cliques by $\widetilde{C}$ and define its membership function, $m_{\widetilde{C}}$, as follows:

Definition 4.1 (Fuzzy Clique) For each node, $x$, we define

$$
m_{\tilde{C}}:= \begin{cases}0 & x \notin \bar{C} \\ 0 \leq p \leq 1 & x \in \bar{C} \\ 1 & x \in \underline{C}\end{cases}
$$

with $p$ denoting the relative frequency of occurrence of node $x$ in $\dot{\bigcup} C\left(t_{i}\right), \dot{\bigcup}$ the disjoint union. Viz.

$$
p:=N(x) / N
$$

$N(x)$ the number of occurrences of $x$ in $\bigcup C\left(t_{i}\right)$.

Remark: We want to remind the reader of the assumptions we have made above. Our fuzzy clique or fuzzy lump is a set with the individual nodes carrying a certain weight, viz. its degree of membership with respect to the fuzzy clique, $\widetilde{C}$, which, on its side, is given by the corresponding membership function, $m_{\widetilde{C}}(x)$. Note that the underlying philosophy is quite modern as it replaces an underlying space (a set of points) by a class of functions on the space. A similar philosophy is e.g. hold in non-commutative geometry and related fields.

We should add at this place some remarks of precaution. We studied in quite some detail in [8] the statistical distribution of the number and size of occurring cliques in, say, a random graph. It turns out that most of the cliques have a typical (generic) size. On the other hand there exists a (possibly small) fraction of degenerated, i.e. very small ones. These small ones may even happen to vanish and/or emerge in the envisaged clock-time interval. That is, we expect our general picture to be true modulo some stochastic noise, which we choose to neglect at the moment. 
Conclusion 4.2 Under the assumption, being made, we can, in a certain approximation, replace the clique graph introduced above by a net of overlapping fuzzy lumps. Mathematically this net is implemented by the corresponding class of membership functions, $\left\{m_{i}(x)\right\}$, with $i$ labelling the fuzzy lumps and where the overlap of cliques is now encoded in the overlap of the supports of the functions, $m_{i}(x)$, viz.

$$
x \in \widetilde{C}_{i} \quad \text { if } \quad m_{i}(x)>0
$$

We see from the above that, to put it briefly, fuzzy-set theory consists of replacing the ground set, $\mathcal{P}(X), X$ some space of points, $\mathcal{P}(X)$ the set of subsets of $X$, and the respective operations on it, by the set of functions, $I^{X}, I$ the unit interval, and corresponding operations on it. $\mathcal{P}(X)$ can be made into a Boolean algebra or a lattice with the help of the operations $\cap, \cup$ or $\cap$ as multiplication and the symmetric difference

$$
\Delta(A, B):=(A \backslash B) \cup(B \backslash A)
$$

as addition.

Corresponding relations can be given for fuzzy sets, but there are a lot of different possibilities to implement or mimik the above set-theoretic operations. The perhaps most straightforward ones are

$$
\cap \rightarrow \min (\widetilde{A}(x), \widetilde{B}(x)) \quad \cup \rightarrow \max (\widetilde{A}(x), \widetilde{B}(x))
$$

where, for brevity, we use from now on the same symbol for the fuzzy set and its membership function. Some more remarks on these matters can be found in the following section. (A nice introduction to fuzzy set theory on a mathematically satisfying level is e.g. [24], including a huge bibliography. A brief but concise representation can also be found in [25]). Some slightly more advanced relations between fuzzy sets, being of potential use in our geometric enterprise are the "degree of being a subset" and the "degree of similarity" between two fuzzy sets. Defining the size of the fuzzy set by

$$
[\widetilde{A}]:=\sum_{x} \widetilde{A}(x) \quad \text { or } \quad \int \widetilde{A}(x) d^{n} x
$$

(existence of the rhs being assumed), we define e.g. the degree of being a subset as

$$
\operatorname{sub}(\widetilde{B} ; \widetilde{A}):=[\widetilde{A} \wedge \widetilde{B}] /[\widetilde{A}]
$$

and the degree of similarity of two sets by

$$
\operatorname{sim}(\widetilde{A}, \widetilde{B}):=[\widetilde{A} \wedge \widetilde{B}] /[\widetilde{A} \vee \widetilde{B}]
$$


with $\wedge, \vee$ extending the notions of $\cap, \cup$; one may take e.g. the above (min, max)implementations.

The above binary relations, given e.g. by ( $\min , \max )$, fullfil the criteria of socalled $(t, s)$-norms (see the next section). There are other such functions in use, which have perhaps nicer properties. One is the so-called Lukasiewicz-(t,s)-norm:

$$
t_{L}(\widetilde{A}, \widetilde{B}):=\max \{0, \widetilde{A}+\widetilde{B}-1\} \quad s_{L}(\widetilde{A}, \widetilde{B}):=\min \{1, \widetilde{A}+\widetilde{B}\}
$$

leading to $\wedge_{L}, \vee_{L}$ (for more details see e.g. [26]). The advantage of the Lukasiewiczoperations is that they induce a metric on fuzzy-sets. We state without proof:

Observation $4.31-\operatorname{sim}_{L}(\widetilde{A}, \widetilde{B})$ defines a metric on fuzzy-sets.

Remark 4.4 We rediscover exactly this t-norm in section (7.9) in quite a different context (E-spaces).

As to this observation, note that for ordinary (finite) sets, $\Delta(A, B)$ induces also a metric.

Observation 4.5 (Hamming-distance) $|\Delta(A, B)|$ defines a metric on finite sets.

Proof: The only non-trivial property is the triangle inequality. As a direct approach is perhaps a little bit tedious, we give a perhaps more pedagogical proof using graph theory. Subsets of a finite set, $X$, can be represented by functions $f \in\{0,1\}^{X}$. These functions, on the other side, can be represented as the vertices of a hypercube, $Q^{|X|} \cdot|\Delta(A, B)|$ is now the minimal length of a path between $f_{A}, f_{B}$ on $Q^{|X|}$, i.e., the minimal number of flips of $(0,1)$ along a path connecting $f_{A}$ and $f_{B}$. This distance on graphs is however a metric.

After this short digression we want to return to our original enterprise, i.e. to relate our approach to the probabilistic-metric-approach, mentioned above. We started from an underlying graph, $G(t)$, and a superimposed clique-graph, $G_{c l}(t)$, both carrying a natural distance function, i.e.

$$
d\left(n_{i}, n_{k}\right) \quad, \quad d_{C}\left(C_{i}, C_{k}\right)
$$

that is, the minimal length of a path, connecting the given nodes (cf. [4] to [8]). Keeping the labelled nodes or cliques (supernodes) fixed, both these distances fluctuate in the course of clock-time evolution as bonds are switched on or off according to one of the microscopic dynamical laws, given above. The cliquemetric will fluctuate since the cliques change their shape and size, viz. also their degree of overlap. On the other side, its fluctuations are supposed to be less 
erratic as a non-void overlap means usually that several nodes and bonds are involved, hence clique-distance should be more stable.

When we switch from this dynamical picture of a time-dependent graph, $G(t)$, to the ensemble picture of fuzzy cliques or lumps, our point of view changes to a static but, on the other side, probabilistic one. This latter point of view is more in the spirit of the framework of Menger et al. That is, the structure of the space under study is no longer time dependent while its largely hidden dynamics is now encoded in various probabilistic notions like e.g. a random metric (which we would like to derive from some underlying principles). We will make the connection to this other framework after the following sections, introducing some technical material about fuzzy-set theory and probabilistic metric spaces. Furthermore we plan to relate in this final section our approach to the complex of ideas developed by e.g. Sorkin et al (see e.g. [29] or [30]; we recommend in particular the latter reference as a thoughtful introduction into this particular bundle of ideas).

\section{Some Concepts from Fuzzy Set Theory}

We give in this section a brief introduction into the ideas of fuzzy set theory. Let $A$ be a fuzzy set on $X$. Then by definition $A(x)$ is interpreted as the degree to which $x$ belongs to $A$. The fuzzy operations like fuzzy unions, intersections and complements are certain (context dependent) generalizations of the corresponding classical set operations. Let e.g. $B$ be another fuzzy set and $\bar{A}$ be the complement of $A$. A particular variant of such fuzzy operations are the following:

$$
\begin{gathered}
\bar{A}(x)=1-A(x) \\
(A \cap B)(x)=\min [A(x), B(x)]
\end{gathered}
$$

and

$$
(A \cup B)(x)=\max [A(x), B(x)]
$$

In the literature, fuzzy intersections and unions are in general defined via socalled $t$-norms (triangular norms) and t-conorms respectively. However, it should be noted that such fuzzy opeartions are by no means unique. Different choices of such t-norms may be appropriate to represent these operations in varying contexts and hence they are known as context dependent operations. Essentially fuzzy set theory provides us with an intutive notion of uncertainty. Subsequent to the development of fuzzy set theory, fuzzy measure theory was developed (see e.g. [27]). The concept of fuzzy measure theory is conceptually an important step 
in understanding the foundational issues related to fuzzy set theory. It provides a broader framework which allows to introduce something like possibility theory.

Crucial in this field as well as in the field of probabilistic metric spaces is the above mentioned notion of $t$-norm (more about the history of this important concept can be found in the book of Schweizer and Sklar, 19 ). The (generalized) intersection of two fuzzy sets $A$ and $B$ is defined by a binary operation on the unit interval as

$$
t:[0,1] \times[0,1] \rightarrow[0,1]
$$

\section{Definition 5.1 (T-Norm)}

$$
\begin{gathered}
t(a, 1)=a \quad \text { (boundary condition) } \\
b \leq d \text { implies } t(a, b) \leq t(a, d) \quad \text { (monotonicity) } \\
t(a, b)=t(b, d) \quad \text { (commutativity) } \\
t(a, t(b, d))=t(t(a, b), d) \quad \text { (associativity) }
\end{gathered}
$$

It is instructive to compare ordinary probability theory with possibility theory and in particular, probability theory with fuzzy set theory. In some sense a fuzzy measure is the dual concept to the concept of fuzzy sets. It encodes the possibility of a given fixed (fuzzy) object to belong to the respective sets of $\mathcal{C}$ (cf. also [28]).

Definition 5.2 Given a set $X$ and a nonempty family $\mathcal{C}$ of subsets of $X$, which, for convenience, we take to be a $\sigma$-algebra. A fuzzy measure on $(X, \mathcal{C})$ is a function $g: \mathcal{C} \rightarrow[0,1]$ that satisfies the following requirements :

1. $g(\emptyset)=0$ and $g(X)=1$ (boundary requirements).

2. for all $A, B \in \mathcal{C}$, if $A \subseteq B$, then $g(A) \leq g(B)$ (monotonicity).

3. for any increasing sequence, $A_{1} \subset A_{2} \subset \cdots$ in $\mathcal{C}$ so that $\bigcup_{i=1}^{\infty} A_{i} \in \mathcal{C}$ we have $\lim _{i \rightarrow \infty} g\left(A_{i}\right)=g\left(\bigcup_{i=1}^{\infty} A_{i}\right)$ (continuity from below)

4. for any decreasing sequence $A_{1} \supset A_{2} \supset \cdots$ in $\mathcal{C}, \lim _{i \rightarrow \infty} g\left(A_{i}\right)=g\left(\bigcap_{i=1}^{\infty}\right)$ (continuity from above).

In case of a probability measure we have for $A \cap B=\emptyset$ :

$$
P(A \cup B)=P(A)+P(B)
$$

From this one sees that ordinary probability measures are a true subclass of possibility or fuzzy measures. As we do not use these more advanced concepts at the moment, we refrain from giving more details which can be found in the mentioned literature. 


\section{Concepts from the Theory of Probabilistic Metric Spaces}

In the statistical geometry, developed by Menger et al, points are no longer considered as the elementary building blocks. In some sense lumps play now their role as primordial, not further resolvable elements. Various concepts of a probabilistic nature are introduced which allow to quantify varying degrees of (in) distinguishability of objects. In this way Menger solved Poincare's dilemma of having, on the one side, a transitive mathematical and, on the other side, a possible intransitive physical relation of equality (cf. [39] and further remarks in section (7). In this geometrical framework we have two basic ingredients:

1. The concept of hazy or fuzzy lumps

2. The "randomisation" of various geometrical or metrical concepts

Frechet ([31]) gave an abstract formulation of the notion of distance in 1906. Hausdorff ([32]) proposed the name metric space and introduced the function $d$ that assigns a nonnegetive real number $d(p, q)$ ( the distance between $\mathrm{p}$ and $\mathrm{q}$ ) to every pair $(p, q)$ of elements (points) of the set $S$. Its properties are

$$
\begin{gathered}
d(p, q)=0 \leftrightarrow p=q \\
d(p, q)=d(q, p) \\
d(p, r) \leq d(p, q)+d(q, r)
\end{gathered}
$$

In 1942 Menger ([33]), guided by the experimental situation in the natural sciences, proposed to replace the "deterministic" function $d(p, q)$ by a more probabilistic concept. He introduced the probability distribution, $F_{p q}$, whose value $F_{p q}(x)$ for any real number $x$, is interpreted as the probability that the distance between $p$ and $q$ is less than $x$.

Remark 6.1 We assume that $F_{p q}$ is continuous from the left. On the other side, we may nevertheless encounter the situation that $F_{p q}^{+}(0)>0$. This means that there may be a non-vanishing probability for two different lumps to have a vanishing distance. This particular point is discussed by Menger in 38 and implements Poincare's observation of a possible non-transitive behavior.

Since probabilities can neither be negative nor be greater than 1, we have

$$
0 \leq F_{p q} \leq 1
$$

for any real $x$. Menger defined a statistical metric space as a set $S$ with an associated set of probability distribution functions $F_{p q}$ which satisfy the following conditions.

$$
F_{p q}(0)=0
$$




$$
\begin{gathered}
\text { If } p=q \text { then } F_{p q}(x)=1 \text { for all } x>0 \\
\text { If } p \neq q \text { then } F_{p q}(x)<1 \text { for some } x>0
\end{gathered}
$$

and

$$
F_{p q}(x+y) \geq T\left(F_{p r}(x), F_{q r}(y)\right)
$$

for all $p, q, r$ in $S$ and all real numbers $x, y$. Here $T$ is a function from the closed unit square $[0,1] \times[0,1]$ into the closed unit interval $[0,1]$ (called a triangular function or triangular norm, see below).

One may view these probabilistic metric spaces as being derived from an underlying fully probabilistic model system, living on a probability space and with the physical observables being random variables. Spacek [34] was the first to look at the subject from this point of view. He proposed the name random metric space instead of probabilistic metric space and discussed the relationship between these two notions. Stevens [35] in his doctoral dissertation tried to modify Spacek's approach. The main idea behind Steven's approach lies in the fact that one has a set $S$ and a collection $P$ of measuring rods. One chooses a measuring rod $d$ from $P$ at random to measure the distance between two given points, $p$ and $q$, of $S$. With the help of this idea Stevens defined the distribution function $F_{p q}$ and showed that the metrically generated space so obtained is a Menger space.

Menger, on the other side, started with a probability distribution function instead of random variables. This is related to the fact that the outcome of any series of measurements of the values of a nondeterministic quantity is a distribution function and the probability space may be unobservable in principle. This point of view has its roots in the positivistic philosophy (cf. e.g. [38]) and is in line with a possible nonclassical behaviour.

Sherwood [36] approached the problem from a different point of view. Following the concept of distribution-generated spaces as introduced by Schweizer and Sklar ( 37]), Sherwood proposed the concept of $E$-space. In an $E$-space, the points are functions from a probability space $(\Omega, \mathcal{A}, P)$ into a metric space $(M, d)$. For each pair, $(p, q)$, of functions in the space, the function $d(p, q)$, defined as

$$
(d(p, q))(\omega)=d(p(\omega), q(\omega))
$$

for all $\omega$ in $\Omega$, is a random variable on $(\Omega, \mathcal{A}, P)$. The function $F_{p q}$ is then the distribution function of this random variable, i.e. we have

$$
F_{p q}(x)=P((d(p, q))(\omega)<x)
$$

In this way, $F_{p q}$ can be regarded as the probability that the distance between $p$ and $q$ is less than $x$. Sherwood showed that every E-space is a Menger space (cf. the subsection (7.2) below). 
Another subclass of probabilistic metric spaces are the so-called distribution generated spaces. The main idea is roughly as follows: Let $S$ be a set. With each point $p$ of $S$ associate an n-dimensional distribution function $G_{p}$ and with each pair, $(p, q)$, a 2 n-distribution function $H_{p q}$ so that it holds:

$$
\begin{aligned}
& H_{p q}(\vec{u}, \vec{v}=(\infty, \ldots \ldots, \infty))=G_{p}(\vec{u}) \\
& H_{p q}(\vec{u}=(\infty, \ldots \ldots, \infty), \vec{v})=G_{q}(\vec{v})
\end{aligned}
$$

for any $\vec{u}=\left(u_{1}, \ldots ., u_{n}\right)$ and $\vec{v}=\left(v_{1}, \ldots \ldots, v_{n}\right)$ in $R^{n}$.

Let now $Z(x)$ be a cylinder in $R^{n}$ with

$$
Z(x):=\left\{(\vec{u}, \vec{v}) \in R^{2 n} ;|\vec{u}-\vec{v}|<x\right\}
$$

for any $x>0$. This defines a distance distribution function, $F_{p q}$, via

$$
F_{p q}(x)=\int_{Z(x)} d H_{p q}=: P_{H_{p q}}(Z(x))
$$

The technical details can be found in e.g. [19]. Note that, in general, the pairs of $S$ need not be independently distributed.

In one interpretation one may view the elements of $S$ as "particles". Then for any Borel set $A$ in $R^{n}$, the integral $\int_{A} d G_{p}$ can be interpreted as the probability that the particle $p$ can be found in the set $A$ and $F_{p q}(x)$ as the probability that the distance between the particles $q$ and $p$ is less than $x$. This then yields another type of probabilistic metric space.

A subclass consists of models where the members in $S$ behave independently of each other. Such spaces are called cloud-spaces(C-spaces). A function $g$ from $R^{n}$ into $R^{+}$is an n-dimensional probability density if the function $G$ defined on $R^{n}$ by

$$
G(\vec{u})=\int_{((-\infty, \ldots \ldots,-\infty), \vec{u}))} g(\vec{v}) d \vec{v}
$$

is an n-dimensional distribution function. If $p$ is a point in a distributiongenerated space over $R^{n}$ such that $G_{p}$ is absolutely continuous, then the corresponding density $g_{p}$ of $G_{p}$ may be visualized as a "cloud" in $R^{n}$ - a cloud whose density at any point of $R^{n}$ measures the relative likelihood of finding the particle $p$ in the vicinity of that point.

Another, equally natural interpretation (which is perhaps more in line with our concept of fuzzy lumps; see section (田)) is to visualize the space as an aggregate of clouds or fuzzy points. To make life simple we may assume that to all points, $p$, is associated a vector, $\vec{c}_{p}$ in $R^{n}$ so that

$$
g_{p}(\vec{u})=g\left(\vec{u}-\vec{c}_{p}\right)
$$


with $g$ sperically symmetric. We may then replace the points by clouds in $R^{n}$ and get a notion of homogeneity. This model is perhaps the most straightforward extension of ordinary (Euclidean) space and resembles our network model of fuzzy lumps (viewed as fuzzy sets) if we chose to embed it into ordinary $R^{n}$.

It can be shown that by taking the convolution product of $g\left(\vec{u}-\vec{c}_{p}\right)$ and $g\left(\vec{v}-\vec{c}_{q}\right)$, one can generate a particular type of random metric space. By applying then a further amount of probabilistic machinery one can create what is called Frechet-Minkowski-metrics, $d_{\beta}$ on the original space $S$, which may e.g. be classical Euclidean space. These metrics are related with and derived from the underlying random metric space (cloud space). The to some extent intricate calculations can be found in [19], chapter 10 .

These Frechet-Minkowski metrics, $d_{\beta}$, associated with (semihomogeneous) $C$ spaces over $R^{n}$ have a remarkable structure. At small distances this metric is non-Euclidean and the distance between two distinct points $p, q$ is bigger than a fixed positive constant, associated with $g(\vec{u})$. On the other hand, it becomes euclidean in the asymptotic region. It appears from the above picture that if we consider a $C$-space as a space of clouds (which may move around), the "haziness" of the distance between $p$ and $q$, which is predominant when their clouds are close together, becomes more and more insignificant when their clouds are sufficiently far apart. In this sense Frechet-Minkowski metrics become asymptotically euclidean.

\section{Random Metrics on our Networks of Fuzzy Lumps}

In this section we want to relate the various complexes of concepts and techniques, developed or presented above, with each other. That is, we view on the one side the (dynamically) fluctuating cliques as smeared out lumps, viz. as fuzzy sets. On the other side, we want to construct so-called probabilistic or random metric spaces over this space of fuzzy lumps.

Note that this task is however not entirely straightforward as within the mathematical framework of the latter approach the underlying space is usually treated as a more or less structureless space of simple points with the underlying possible causes of the fluctuations in the metrical distance usually not being openly discussed. This has however to be done in the concretely given model systems.

We should ad the remark that there are also different scientific philosophies behind these various points of view (cf. [19 p.17). There exists a tradition to keep the (or a) underlying concrete probability space out of the game and regard is as secondary, while concentrating on the class of (phenomenologically given) distribution functions.

On the other side, a concretely given (and, what is even better, physically 
motivated) microscopic probabilistic ground space provides (among other things) a common and unifying reference frame. In other words, we have a canonical deterministic metric on the underlying dynamical graph, $G(t)$ or its clique graph, $G_{c l}(t)($ cf. (7.2)). We have argued that our cliques or lumps are fluctuating as a result of the imposed dynamical laws (discussed in section 2). From this input we have to infer the concept of a probabilistic distance between two given labelled (fuzzy) lumps. We divide our section into two subsections. In the first subsection we introduce distance concepts on the space of overlapping (static) fuzzy lumps as they are given in the context of fuzzy set theory as discussed in section 4 . In this context fluctuations are only implicitly included and the model theory has (one may say) the status of kind of a mean field theory. On the other side, the metrics, we introduce in the following subsection are related in spirit to distance concepts introduced by Menger in his interesting essay [38]. More fundamental are in our view the concepts we develop in the other subsection. We construct an explicit probability space, introduce metrical distance as an explicitly given random variable, find the corresponding triangular norm and show that the space we get is a model of a so-called E-space, introduced in section 6. These E-spaces are a subclass of Mengers probabilistic metric spaces.

\subsection{Metrics on Fuzzy Lumps, the Mean Field Picture}

The perhaps most immediate method to impose some metric distance on our space of fuzzy lumps is the following one. In a first step we define a distance concept for the immediate ("infinitesinal") neighborhood of a given lump and then proceed (for finite distances) by a chain method, viz. paste infinitesimal distances together.

We start with two fuzzy lumps, $\widetilde{C}_{0}, \widetilde{C}_{1}$, which overlap as fuzzy sets. We hence have (see section 4):

$$
0 \leq p\left(\widetilde{C}_{0}, \widetilde{C}_{1}\right):=1-\operatorname{sim}\left(\widetilde{C}_{0}, \widetilde{C}_{1}\right)<1
$$

We have now several possibilities to proceed. We can e.g. fix a certain scale resolution and state that two fuzzy lumps are indistinguishable if

$$
p\left(\widetilde{C}_{0}, \widetilde{C}_{1}\right) \leq \varepsilon
$$

This is in accord with already classical ideas of Poincaré, developed in [39, p.31f. Put differently, in the physical world we may have a non-transitive infinitesimal distance concept or, more generally, a non-transitive concept of identity so that

$$
A=B, B=C, A \neq C
$$

or

$$
d(A, B)=0, d(B, C)=0, d(A, C) \neq 0
$$


Definition 7.1 (Distance, Variant 1) Denoting by $\gamma$ a path connecting $\widetilde{C}_{i}$ and $\widetilde{C}_{j}$, which consists of a chain s.t.

$$
\widetilde{C}_{i}=: \widetilde{C}_{k_{0}}, \widetilde{C}_{j}=: \widetilde{C}_{k_{n}}
$$

and

$$
p\left(\widetilde{C}_{k_{l}}, \widetilde{C}_{k_{l+1}}\right) \leq \varepsilon
$$

we define

$$
\operatorname{dist}_{\varepsilon}\left(\widetilde{C}_{i}, \widetilde{C}_{j}\right):=\inf _{\gamma} n
$$

( dist $_{\varepsilon}:=\infty$ if there is no such chain).

It can be easily shown that this definition fullfils the axioms of a metric. Note however that the validity of the triangle inequality is enforced by attributing the distance 'one' to two "indistinguishable" lumps. This is a little bit unpleasant but inescapable if we want to base our macroscopic distance concept on the concatenation of "infinitesimal" distances. On the other side, this kind of distance is probabilistic in a very restricted sense and yields only distances which are multiples of a fixed given distance element.

We can improve the situation by taking as infinitesimal distance elements the above measures of overlap, $p\left(\widetilde{C}_{k_{l}}, \widetilde{C}_{k_{l+1}}\right)$, and define

\section{Definition 7.2 (Distance, Variant 2)}

$$
d\left(\widetilde{C}_{i}, \widetilde{C}_{j}\right):=\inf _{\gamma} \sum p\left(\widetilde{C}_{k_{l}}, \widetilde{C}_{k_{l+1}}\right)
$$

Note that in contrast to the preceding definition now the infimum need not be taken at a path of minimal canonical length. Quite the contrary, in exceptional situations the canonical length of a path leading to a very short distance may be quite large, whereas this is not expected to be the ordinary situation. Anyway, the second distance concept is a little bit more probabilistic.

\subsection{The Space of Cliques as a Probability Space and the Connection with Random Metric Spaces}

We repeat briefly what we have said in preceding sections. We have assumed that we have a network of a more or less fixed number of, however, fluctuating cliques. That is, their degree of overlap may vary in the course of time. At each discrete time step during a sufficiently long time interval, $I$, (so that we can exploit probabilistic concepts in, at least, a certain approximation) we have 
a definite array of overlapping cliques, $G_{c l}(t)$. As ground set for our probability space we take this set, i.e.

$$
X:=\left\{G_{c l}(t), t \in I,|I|=N, N \text { some sufficiently large number }\right\}
$$

The simplest assumption is to assume that all configurations have the same probability, $P\left(G_{c l}(t)\right):=1 / N$, say.

Remark 7.3 On physical grounds a more sophisticated probability density may suggest itself. Our results, derived below, are however independent of the particular choice of $P$.

Observation 7.4 The above defines a discrete probability space, $X$, on which random variables can be introduced, their expectation value being denoted by $<>$.

Example 7.5 We previously introduced the membership function, $\widetilde{C}_{i}(x)$, of a fuzzy lump, $\widetilde{C}_{i}$. Defining the elementary random variable ( $x$ a node of the primordial underlying graph)

$$
x_{C_{i}}\left(G_{c l}(t)\right)= \begin{cases}1 & x \in C_{i}(t) \\ 0 & \text { else }\end{cases}
$$

we have

$$
\widetilde{C}_{i}(x)=\left\langle x_{C_{i}}\right\rangle=: x\left(\widetilde{C}_{i}\right)
$$

(cf. the section about fuzzy lumps).

In the same sense we can define the distance between two fixed lumps, $C_{i}, C_{j}$, as a random variable on our probability space, taking the values $d_{\left(C_{i}, C_{j}\right)}\left(G_{c l}(t)\right)$. In contrast to our ordinary fuzzy lumps, all these random variables do fluctuate, the fluctuations given by

$$
\left\langle\left(x_{C_{i}}-\left\langle x_{C_{i}}\right\rangle\right)^{2}\right\rangle \quad \text { or } \quad\left\langle(d-\langle d\rangle)^{2}\right\rangle
$$

For simplicity reasons we write from now on the metric as $d(p, q), p, q$ denoting some cliques, and with the understanding that it represents the above random variable.

If we want to relate our approach to the probabilistic metric framework laid out in e.g. [19, we have to inspect the transitivity properties of our distance concept. 
Observation 7.6 With p, q,r three points (cliques), we have for the respective random variables

$$
d_{p, r} \leq d_{p, q}+d_{q, r}
$$

as they are evaluated on a fixed network, $G_{c l}(t)$, where the ordinary triangle inequality of the canonical graph distance holds. Hence

$$
\left\langle d_{p, r}\right\rangle \leq\left\langle d_{p, q\rangle}+\left\langle d_{q, r}\right\rangle\right.
$$

On the other side, probabilistic metric spaces are defined via the probability distributions, $F_{p q}(x)$, denoting the probability that the distance between $p, q$ is smaller than $x$. The ordinary triangle inequality is replaced in this framework by a more complicated estimate (cf. section 6):

$$
F_{p r}(x+y) \geq T\left(F_{p q}, F_{q r}\right)
$$

with $T$ a triangular norm.

Our task consists now in determining this function $T$ within our own stochastic framework. To this end we employ the following formula which holds for probability measures (i.e. $P(X)=1$ ).

Lemma 7.7 With $A, B$ measurable sets in $X$ it holds

$$
P(A \cap B) \geq P(A)+P(B)-1
$$

Proof: This result has also been employed in [19], p.26. For completeness sake we repeat it here. We have

$$
\begin{aligned}
P(A \cap B)=P(A)+P(B)-P(A \cup B) \geq P(A)+P(B)-P(X) & =P(A)+P(B)-1
\end{aligned}
$$

and therefore

$$
P(A \cap B) \geq \max (P(A)+P(B)-1,0)
$$

This proves the lemma.

We have by definition:

$$
F_{p r}(x+y)=P\left(d_{p r}<x+y\right)
$$

which, for the simplest choice of $P$, is just the fraction of configurations, $G_{c l}(t)$, with the canonical graph metric fulfilling this inequality. As the triangle inequality holds for the canonical graph metric we have

$$
P\left(d_{p q}<x, d_{q r}<y\right) \leq P\left(d_{p r}<x+y\right)
$$

and hence, with the help of the above lemma:

$$
F_{p r}(x+y)=P\left(d_{p r}<x+y\right) \geq \max \left(F_{p q}(x)+F_{q r}(y)-1,0\right)
$$


Observation 7.8 We proved that our random metric, introduced above, fulfills a triangular inequality

$$
F_{p r}(x+y) \geq W\left(F_{p q}(x), F_{q r}(y)\right)
$$

with $W$ the triangular norm

$$
W(a, b):=\max (a+b-1,0)
$$

Hence our above probability space is a Menger space.

(That $W$ is in fact a triangular norm is easy to show and is also employed in e.g. [19]).

A special class of probabilistic metric spaces are the so-called E-spaces (cf. the section [6 and [19], chapt. 9). To complete our analysis we can reframe our model so that it becomes such an E-space. The ground space, $X$, is our probability space. The metric target space, $M$, is a new space-time supergraph, defined as follows:

\section{Definition 7.9 (Space-Time Picture of Clique Graph)}

$$
M:=\bigcup_{t \in I} G_{c l}(t)
$$

with the understanding that exactly the nodes $C_{i}(t)$ and $C_{i}(t+1), t, t+1 \in I$, are connected by additional bonds. That is, $C_{i}(t)$ describes the orbit of the fixed but fluctuating clique, $C_{i}$, in the course of time.

We can now relate bijectively the cliques, $C_{i}$, and certain functions from $X$ to $M$.

Observation 7.10 With the class of functions, $C_{i}$,

$$
C_{i}\left(G_{c l}(t)\right):=C_{i}(t) \in M
$$

and their distance

$$
d_{p q}\left(G_{c l}(t)\right):=d\left(C_{p}(t), C_{q}(t)\right)
$$

the above probability space can be considered as an E-space.

\section{References}

[1] L.Smolin: "The Future of Spin Networks", gr-qc/9702030

[2] C.Rovelli: "Loop Quantum Gravity", gr-qc/9710008 
[3] C.J.Isham: "Structural Issues in Quantum Gravity", gr-qc/9510063 (Lecture given at the GR14 Conference, Florence 1995)

[4] T.Nowotny, M.Requardt: "Dimension Theory on Graphs and Networks", J.Phys.A:Math.Gen. 31(1998)2447, hep-th/9707082

[5] T.Nowotny, M.Requardt: "Pregeometric Concepts on Graphs and Cellular Networks", invited paper J.Chaos, Solitons and Fractals 10(1999)469, hep-th/9801199

[6] M.Requardt: "Cellular Networks as Models for Planck-Scale Physics", J.Phys.A:Math.Gen. 31(1998)7997, hep-th/9806135

[7] M.Requardt: "Spectral Analysis and Operator Theory on (Infinite) Graphs ... ", submitted to JPA, math-ph/0001026

[8] M.Requardt: "(Quantum) Space-Time as a Statistical Geometry of Lumps in Random Networks", Class.Quant.Grav. 17(2000)2029, gr-qc/9912059

[9] C.J.Isham: "Canonical Quantum Gravity and the Problem of Time", Lectures presented at the NATO Advanced Study Inst. "Recent Problems in Math.Phys.", Salamanca June 1992, gr-qc/9210011

[10] J.Butterfield, C.J.Isham: "On the Emergence of Time in Q.Gr.", gr-qc/9901024 to appear in "The Argument of Time", ed.J.Butterfield, Oxford Univ.Pr., Oxford 1999

[11] M.Requardt: "Let's Call it Nonlocal Quantum Physics", Goettingen Preprint, gr-qc/0006063

[12] Th.Nowotny: Diploma Thesis, a compilation of some of the extensive computer simulations can be found on the web site: http://www.physik.unileipzig.de/ nowotny/research.htm] (commentary in german)

[13] T.Toffoli, N.Margolus: "Cellular Automaton Machines", MIT Pr., Cambridge Mass. 1987

[14] F.Markopoulou, L.Smolin: "Causal Evolution of Spin Networks", Nucl.Phys. B508(1997)409 or gr-qc/9702025,

F.Markopoulou: "Dual Formulation of Spin Network Evolution", gr-qc/9704013

[15] R.Borissov: "Graphical Evolution of Spin Network States", Phys.Rev. D55(1997)6099 or gr-qc/9606013

[16] K.Menger: in "Albert Einstein Philosopher Scientist", ed. P.A.Schilpp, 3rd edition Cambridge Univ.Pr., London 1970

[17] N.Rosen: "Statistical Geometry and Fundamental Particles", Phys.Rev. $72(1947) 298$ 
[18] S.Roy:"Statistical Geometry and Applications to Microphysics and Cosmology", Kluver Acad.Publ. Dordrecht 1998

[19] B.Schweizer,A.Sklar:"Probabilistic Metric Spaces", North-Holland N.Y. 1983

[20] J.Stachel: "Einstein and Quantum Mechanics" in "Conceptual Problems of Quantum Gravity", eds. A.Asthekar,J.Stachel, Einstein Studies vol.2, Birkhäuser Boston 1991

[21] C.J.Isham, Y.Kabyshin, P.Renteln: "Quantum Norm Theory and the Quantisation of Metric Topology", Class.Quant.Grav. 7(1990)1053

[22] C.J.Isham: "An Introduction to General Topology and Quantum Topology", Lectures presented at the Advanced Summer Institute on Physics, Geometry and Topology, Banff August 1989

[23] P.Alexandroff, H.Hopf: "Topologie” (reprint), Springer N.Y. 1974

[24] R.Lowen: "Fuzzy Set Theory", Kluwer, Dordrecht 1996

[25] "I.N.Bronstein,K.A.Semendjajew": "Taschenbuch der Mathematik", Harry Deutsch, Frankfurt 1997

[26] B.Demant: "Fuzzy-Theorie, Vieweg, Braunschweig 1993

[27] Z.Wang, J.H.Klir: "Fuzzy Measure Theory", Plenum Pr. N.Y. 1992

[28] H.B.Bandemer, S.Gottwald: "Einfuehrung in Fuzzy-Methoden", Akademie Verlag, Berlin 1993

[29] L.Bombelli,J.Lee,D.Meyer,R.Sorkin: "Space-Time as a Causal Set", Phys.Rev.Lett. 59(1987)521

[30] R.Sorkin: "A Specimen of Theory Construction from Quantum Gravity" which appeared in "The Creation of Ideas in Physics", ed. J.Leplin, Kluwer, Dordrecht 1995, gr-qc/9511063

[31] M.Frechet: "sur quelques points du calcul fonctionnel", Rend.Circ.Mat.Palermo $22(1906) 1$.

[32] F.Hausdorff: "Grundzuge der Mengenlehre" Leipzig, Veit und Comp(1914).

[33] K.Menger: "Statistical Metrics", Procd. Nat.Acad.Sci.USA,28(1942)535.

[34] A.Spacek: "Note on Menger's Probabilistic Geometry", Czechoslovak.Math.Journ. 6(1956)72

[35] R.R.Stevens: "Metrically generated probabilistic metric spaces", Fund.Math.61(1968)259 
[36] H.Sherwood: "On E-spaces and their relation to other classes of probabilistic metric spaces", J.London.math.Soc. 44(1969)441.

[37] B.Schweizer,A.Sklar: "Statistical Metric Spaces", Pacific J.Math.,10(1960)313.

[38] K.Menger: "Geometry and Positivism, a Probabilistic Microgeometry", in K.Menger, Selected Papers in Logic and Foundation ... p.225, Ed. H.L.Mulder, Reidel Publ.Comp., Dordrecht 1979

[39] H.Poincaré: "Science and Hypothesis", Dover Pub., N.Y. 1952 